Old Dominion University

ODU Digital Commons

Physics Faculty Publications

Physics

4-1996

\title{
Ionization of Rydberg Wave Packets by Subpicosecond, Half-Cycle Electromagnetic Pulses
}

C. Raman

C.W.S. Conover

C. I. Sukenik

Old Dominion University, csukenik@odu.edu

P. H. Bucksbaum

Follow this and additional works at: https://digitalcommons.odu.edu/physics_fac_pubs

Part of the Atomic, Molecular and Optical Physics Commons

\section{Repository Citation}

Raman, C.; Conover, C. W. S.; Sukenik, C. I.; and Bucksbaum, P. H., "Ionization of Rydberg Wave Packets by Subpicosecond, HalfCycle Electromagnetic Pulses" (1996). Physics Faculty Publications. 8.

https://digitalcommons.odu.edu/physics_fac_pubs/8

\section{Original Publication Citation}

Raman, C., Conover, C.W.S., Sukenik, C.I., \& Bucksbaum, P.H. (1996). Ionization of Rydberg wave packets by subpicosecond, halfcycle electromagnetic pulses. Physical Review Letters, 76(14), 2436-2439. doi: 10.1103/PhysRevLett.76.2436

This Article is brought to you for free and open access by the Physics at ODU Digital Commons. It has been accepted for inclusion in Physics Faculty Publications by an authorized administrator of ODU Digital Commons. For more information, please contact digitalcommons@odu.edu. 


\title{
Ionization of Rydberg Wave Packets by Subpicosecond, Half-Cycle Electromagnetic Pulses
}

\author{
C. Raman, C. W. S. Conover,* C. I. Sukenik, and P. H. Bucksbaum \\ Department of Physics, University of Michigan, Ann Arbor, Michigan 48109-1120
}

(Received 19 May 1995; revised manuscript received 6 November 1995)

\begin{abstract}
We have studied the ionization of Rydberg wave packets by subpicosecond, nearly unipolar electromagnetic field pulses, in the regime where the duration of the electric field is less than the classical Kepler orbit time $2 \pi \bar{n}^{3}$ for the wave packet. In contrast to the subpicosecond optical pulses, subpicosecond field pulses can ionize wave packets when the probability density near the inner turning point of the Kepler orbit is low. The transfer of energy from the electromagnetic field to essentially free electrons demonstrates that the pulses are substantially shorter than one field cycle. Such "half-cycle" pulses can track the wave packet throughout its orbit, in order to study wave packet trajectories or other processes at the quantum-classical boundary.

PACS numbers: $32.80 . \mathrm{Rm}, 03.65 . \mathrm{Sq}$
\end{abstract}

High-power, subpicosecond electromagnetic field pulses [1] are a new tool for studying dynamics in weakly bound systems [2]. These bursts of coherent radiation have peak powers up to $1 \mathrm{MW}$ and coherent bandwidths of several THz, centered at $0.5 \mathrm{THz}[1]$. Original studies of the scaling behavior for $\mathrm{THz}$ field ionization, together with more recent studies of the ionization of Rydberg Stark states, have provided indirect evidence that these pulses are nearly unipolar (i.e., "half" of an optical cycle) [3]. This unusual field shape gives rise to interactions with atoms that are not possible using conventional microwave pulses or light pulses.

Some classical analyses have been successful in describing the scaling laws observed in ionization of Rydberg eigenstates or Stark eigenstates using half-cycle pulses (HCP). This close correspondence to classical ideas about trajectories of electrons in Kepler orbits motivated us to study HCP interactions with nonstationary states as well. We are particularly interested in states that closely resemble their classical counterparts, such as localized Rydberg wave packets. This Letter reports our findings.

Our principal observation is that the HCP can ionize a radially localized Rydberg wave packet over its entire trajectory, and not just at its inner turning point. This is quite different from a short optical pulse, where the ionization cross section vanishes if the wave packet is far from the inner orbital turning point [4]. The amount of ionization as a function of peak HCP field is dramatically different for wave packets close to the core vs wave packets near the outer turning point. These results are consistent with model calculations of a sudden impulse imposed on a radial wave packet. We conclude that HCP ionization offer a means of tracing wave packet trajectories around a bound quantum system. This is a useful tool in studies of quantum wave packet dynamics.

The Rydberg wave packets in this experiment are cesium $p$ states with principal quantum numbers in the range $24<n<30$ and corresponding classical orbit times from 1.3 to $2.8 \mathrm{ps}$. The wave packets are produced as follows: Cesium atoms in an effusive thermal beam are first excited from the $6 \mathrm{~s}$ ground state to the $7 \mathrm{~s}$ state via two-photon excitation, using weakly focused, $10 \mathrm{~ns}, 1079 \mathrm{~nm}$ laser pulses produced by Raman shifting a $745.0 \mathrm{~nm}$ dye laser in a pressurized hydrogen cell. The Raman-shifted light intersects the Cs beam between two capacitor plates in a vacuum chamber. Approximately $5 \mathrm{~ns}$ after excitation, the Cs atoms are further excited from the $7 s$ state into $n p$ Rydberg states by illumination with a linearly polarized $790 \mathrm{~nm} 100 \mathrm{fs}$ pulse. The wave packet then evolves for a time $\tau$, after which it is further excited by a HCP. Typically, this produces partial ionization, and partial redistribution into other bound states.

Prompt ionization and redistribution into higher bound states by the HCP can each be detected. A small electric field $(<100 \mathrm{~V} / \mathrm{cm})$ accelerates either electrons or ions through a small hole $(\sim 2 \mathrm{~mm}$ diameter $)$ in the top capacitor plate to a channel-plate detector. Following this, a larger field (up to $5 \mathrm{kV} / \mathrm{cm}$ ) is slowly ramped up to ionize the redistributed bound-state Rydberg population, using the same detector. The slow ramp ionizes the more weakly bound states first. (The ionization field required is inversely proportional to the square of the binding energy.) This permits separate detection of each bound state over much of the range of redistribution. Furthermore, if the HCP is blocked, this same detection scheme permits us to analyze the state content of the wave packet excited by the optical pulse.

The spectral content of such a wave packet has previously been observed using an optical version of the Ramsey method of separated oscillatory fields [5,6]. This technique produces a signal which is proportional to the wave packet temporal autocorrelation function $S(t)=$ $|\langle\psi(\mathbf{r}, t) \mid \psi(\mathbf{r}, 0)\rangle|$. The Fourier transform of this function gives spectral information about the wave packet; however, in the limit of weak excitation, there is no information about the relative phases of the component eigenstates. In other words, there is no information about the 
shape of the wave packet, and spatially dispersed wave packets will give precisely the same autocorrelation as compact ones [7]. Optical cross correlations have been used to obtain more specific information about the overlap of a wave packet with the atomic core [8].

More detailed information about the motion of the wave packet to the outer parts of its range is very difficult to obtain using optical pulses as a probe. The cross section for optical excitation or ionization of a wave packet is dramatically reduced as the packet moves away from the core. This is simply a consequence of momentum conservation, which forbids inelastic scattering between free electrons and single photons. Our unipolar field pulses overcome this problem. Since a HCP has a nonzero electric field integral, it can exert a nonzero force on a free electron. Therefore we have studied the ionization of the radial wave packets described above with HCPs.

The HCP used in this study has been described in detail elsewhere. Briefly, it is a nearly unipolar pulse with a ratio of positive to negative field amplitudes of approximately $5: 1$. The duration of the positive loop is approximately $400 \mathrm{fs}$ full width at half maximum. The pulse is produced by a $3.5 \mathrm{~cm} \times 3.5 \mathrm{~cm}$ dc-biased GaAs photoconducting switch illuminated by a $100 \mathrm{fs}$ laser pulse, derived from the same laser that excites the Cs wave packet. The electric field autocorrelation, spectrum, and approximate pulse shape are discussed in detail in Ref. [1].

In practice, there is often a second, smaller unipolar pulse produced due to reflection from the back surface of the GaAs wafer [9]. This was undesirable for this experiment. We discovered that this postpulse could be essentially eliminated by using the HCP produced in reflection from the GaAs wafer, rather than the transmitted HCP. Apparently in this case, the reflection from the wafer's back surface is severely attenuated by the electron-hole plasma created by the laser on the front surface and the effect of the post pulse is minimized.

The HCP was reflected from an off-axis gold paraboloid mirror into the interaction region, along the same direction as the optical pulse that formed the wave packet. A thin dielectric reflector for the optical pulse served as the beam-combining element, ensuring that both beams propagate collinearly. The polarization of the HCP was either aligned or crossed with that of the optical pulse.

To calibrate the HCP peak field in the interaction region, we excited $n=25 p$ states in Cs with a tunable dye laser, and ionized them with the HCP. Then, using the analysis described in Ref. [2], we calibrated the field necessary for the first appearance of ionization. Because of the half-cycle nature of the pulse, full ionization of the state is possible despite the fact that the duration is much less than the Kepler orbit time.

Figure 1 shows ionization versus time delay between the HCP and the exciting pulse, for a wave packet with

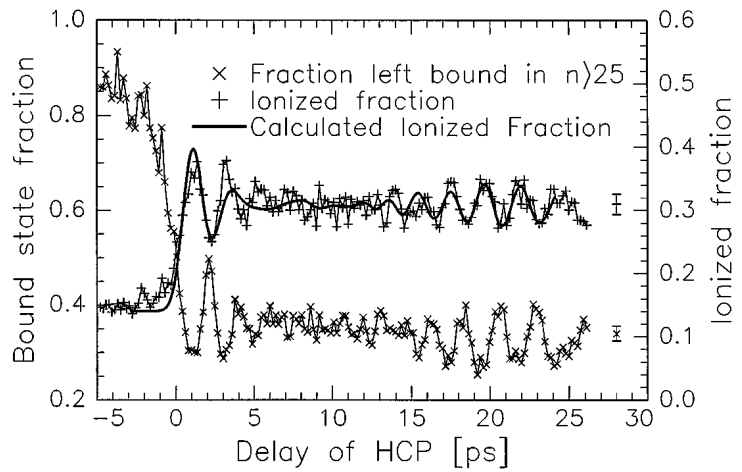

FIG. 1. Fraction of atoms ionized by HCP (+) as well as the fraction left in bound states with $n>25(\times)$, as a function of time delay, showing the partial revival of the wave packet. The HCP had a peak field of $6.3 \mathrm{kV} / \mathrm{cm}$, and a duration of approximately $400 \mathrm{fs}$. The bold curve is the result of a onedimensional calculation described in the text, for a momentum transfer of 0.02 atomic unit.

a Kepler orbit time of 2 ps. The error bars are statistical, based on measured signal fluctuations when the time delay is kept fixed. The polarization of the HCP is orthogonal to that of the optical pulse. The peak field, calibrated as described above, was $6.3 \mathrm{kV} / \mathrm{cm}$. At the earliest time delays the HCP arrives before the wave packet is created and no ionization occurs. Nonetheless, some ionization signal is always detected at negative time delays for various reasons: There is a long low-amplitude trailing tail on the HCP that can interact with the wave packet at negative time delays; there may be some residual reflected pulse as well; and some multiphoton ionization by either the $1.08 \mu \mathrm{m}$ pulse or the $790 \mathrm{~nm}$ pulse can occur. The ionization signal first increases as both the exciting pulse and the HCP become coincident. It reaches a peak and subsequently begins to decrease, indicating that the wave packet has moved away from the core region and its momentum has diminished substantially. Finally, we observe a wave packet's return to the core one Kepler orbit later, corresponding to a time of roughly $2 \mathrm{ps}$. The wave packet disperses in the Coulomb potential, and then revives in about $15 \mathrm{ps}$ [8]. Oscillations in the ionization rate are damped by dispersion, and reappear as the wave packet reassembles during its revival.

A second curve in Fig. 1 shows the fraction of bound state population left after the passage of the HCP, for states above $n=25$. These data were obtained by ramped field ionization, as described above. This curve oscillates out of phase with the ionization signal, and shows the same dispersion and revivals. A third curve shows the results of a calculation, based on the model described below, of the ionization versus time delay. To perform this calculation, we measured the amplitude of the different eigenstates making up the wave packet by field ionizing them in the same apparatus. This information was used to construct a model wave packet. 


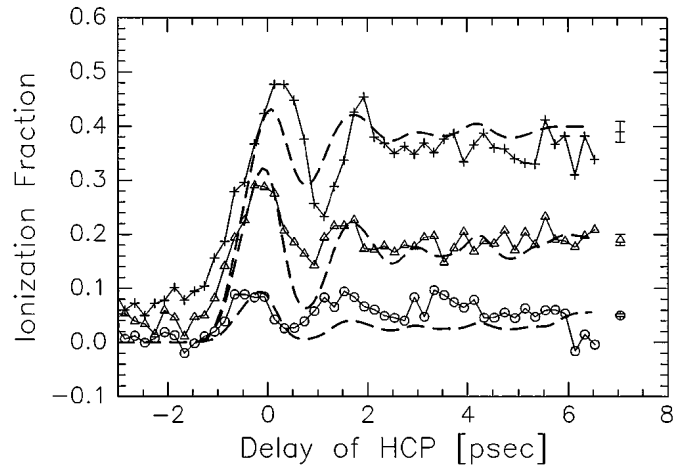

FIG. 2. Fraction of atoms ionized by HCP vs time delay at peak fields of $3.1 \mathrm{kV} / \mathrm{cm}$ (bottom), $4.6 \mathrm{kV} / \mathrm{cm}$ (middle), and $7.1 \mathrm{kV} / \mathrm{cm}$ (top). The dashed curves are the results of the onedimensional calculation described in the text, with impulses of 0.02 (bottom), 0.03 (middle), and 0.04 (top) atomic unit.

Figure 2 shows the ionization of the wave packet versus time delay during the first few Kepler orbit times, for three different magnitudes of the electric field in the interaction region, for a slightly different wave packet with Kepler orbit time of $1.6 \mathrm{ps}$. Here the polarizations of the two pulses are parallel. For these $n p$ states, the polarization of the $\mathrm{THz}$ pulse with respect to the optical pulse does not affect the essential character of the oscillations, dispersion, and revival. Note that these more deeply bound wave packets suffer substantially less ionization for negative time delays. The dispersion is greater here, but we can again detect the evolution of the wave packet through one full Kepler cycle before dispersion breaks it up and spreads the probability throughout the atom. Again, the calculations are based on model wave packets, ionized according to our model described below.

Figure 3 shows the ionization of the wave packet from Fig. 2 as a function of HCP field, at two time delays, corresponding to the maximum and minimum signal in the wave packet evolution. This corresponds to time $t=0.2 \mathrm{ps}$ in Fig. 2, when the wave packet is localized

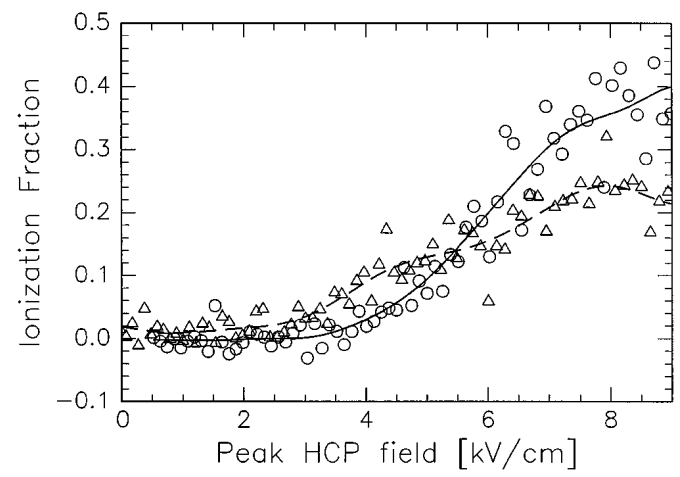

FIG. 3. Ionization of the wave packet $(A)$ near the core (circles) and $(B)$ near the outer turning point (triangles), as a function of peak field in the HCP. The solid lines are the same data after smoothing. near the core, and $t=1 \mathrm{ps}$, when the wave packet is near its outer turning point. Although the two curves begin rising at about the same field, their shape is quite different for ionization below 50\%. Beyond this, the curves are expected to approach one another, since at very high peak fields in the HCP the wave packet can be ionized anywhere in its orbit.

We can analyze these results in light of our previous knowledge of the interaction between Rydberg states and the HCP. There we found that the HCP can fully ionize Rydberg states even if they have corresponding classical orbit times far longer than the pulse duration [2]. Furthermore, we found that atoms with permanent dipole moments (i.e., Stark states) ionize at different HCP fields depending on whether the HCP is polarized along or against the atomic dipole direction. Both of these results agree with semiclassical analyses that required the halfcycle nature of the pulse, and both results also require that the HCP ionize electrons that are near their outer turning points. Now, with wave packets, we are in a position to observe this phenomenon directly.

Curve $A$ in Fig. 3 corresponds to ionizing a wave packet localized near the core of the atom. Here the momentum is already quite high in the atom, so we expect that it should not take as much additional momentum transfer from the HCP to increase the electron energy to escape the potential. Curve $B$ corresponds to a wave packet localized near the outer turning point of the atom. Since it is almost at rest, and nearly free, the HCP must provide nearly all the momentum to ionize such a wave packet. Curve $B$ does rise more slowly than curve $A$ on average, but there is significant additional structure, particularly near the onset of ionization that departs from simple classical behavior.

To gain some understanding of these data, we have performed a simple model calculation. We begin with the eigenvalue equation for a single electron in a Coulomb potential (atomic units will be used throughout):

$$
\left[-\frac{1}{2} \frac{\partial}{\partial r^{2}}+\frac{l(l+1)}{2 r^{2}}-\frac{1}{r}\right] \chi(r)=E \chi(r) .
$$

When $E$ is the energy of a stationary Rydberg state, this is the equation used to compute the alkali radial wave functions in single-channel quantum defect theory [10]. For our problem we will use for $E$ the weighted distribution of energies of the $n p$ states in the Cs wave packet, and interpret this equation as the energy functional for the wave packet. In other words, the first term on the left hand side is the local kinetic energy of the wave function $T(r)$, the other terms make up the potential energy $V(r)$, and $T(r)=E(r)-V(r)$. The local wavemechanical radial momentum is just $p(r)= \pm \sqrt{2 T(r)}$. The sign depends on initial conditions.

Our wave packets are $p$ states with $l=1, m_{l}=0$, so they are not really one-dimensional objects. Nonetheless, for the purposes of this calculation, we will only consider 
motion along $\hat{z}$, the direction of the applied HCP. If the HCP is short in duration compared to the motion of the wave packet, then its effect is to impart an impulse to the electron. The initial wave momentum $p_{z}(z)$ is just $p(r)$ on one side of the origin, and $-p(r)$ on the other side. After the HCP passes, $p_{z}(z) \rightarrow p_{z}(z)+p^{\prime}$, where

$$
p^{\prime}=\int_{-\infty}^{\infty} F(t) d t
$$

This impulse changes the kinetic energy:

$$
T(z) \rightarrow T^{\prime}(z)=\frac{\left[p_{z}(z)+p^{\prime}\right]^{2}}{2} .
$$

The energy is also transformed: $E \rightarrow E^{\prime}(z)=T^{\prime}(z)+$ $V(z)$. If $E(z)$ at a particular position becomes greater than zero, we will say that part of the wave packet becomes ionized.

To compare this calculation to experiment, we used single-channel quantum defect wave functions to build a wave packet similar to the one created in the experiment. The impulse ionization curves were then convolved with a 400 fs Gaussian to simulate the convolution of the wave packet with the HCP.

Results of our calculation of the ionization of the wave packet as a function of time delay are shown in Figs. 1 and 2. The momentum transfers were chosen such that the average ionization rate (i.e., after the oscillations have died out, at about $6 \mathrm{ps}$ after the wave packet is created) matched the data, excluding the small amount of ionization that occurs at negative time delays. The impulse imparted depends sensitively on the duration of the HCP, which we know only approximately. Moreover, although we are able to calibrate the electric field used in the experiment by comparing it to the HCP field required to ionize the $n=25 p$ Rydberg state, there remains a residual uncertainty in that absolute electric field value [2,9]; however, a precise knowledge of the electric field (and, hence, the absolute momentum transfer to the atom) is not necessary to demonstrate that our model contains the essential physics. The position of the oscillations in Figs. 1 and 2 and the relative ionization fraction at the peak of the pulse and at the outer turning point are approximately described by our impulse model. Full quantum-mechanical calculations are the only way to include the important effects of the finite duration of the HCP and the three-dimensional character of the wave packet.

In conclusion, we have observed the ionization of a radial Rydberg wave packet in Cs by a half-cycle pulse, as the wave packet orbits the atom. In contrast to previous techniques, HCP ionization is capable of detecting wave packet motion anywhere in the atom, not just near an ion core. We would like to apply this technique to more complex systems where the wave packet motion away from the core reveals interesting physics. Two examples are molecules and atoms in strong external fields.

We wish to acknowledge many useful discussions with Robert Jones, Douglass Schumacher, and Donne You. This work was supported by the National Science Foundation. One of us (C. W. S. C.) received support from the Fellow Program of the Michigan Center for Ultrafast Optical Science, an NSF Science and Technology Center.

*On sabbatical from Colby College, Waterville, Maine.

[1] D. You, R. R. Jones, D. R. Dykaar, and P. H. Bucksbaum, Opt. Lett. 18, 290 (1993); B. I. Greene, J. F. Federici, D. R. Dykaar, R. R. Jones, and P.H. Bucksbaum, Appl. Phys. Lett. 59, 893 (1991).

[2] R. R. Jones, D. You, and P.H. Bucksbaum, Phys. Rev. Lett. 70, 1236 (1993).

[3] R. R. Jones, N.E. Tielking, D. You, C. Raman, and P. H. Bucksbaum, Phys. Rev. A 51, R2687 (1995).

[4] A. ten Wolde, L.D. Noordam, A. Ligendijk, and H. B. van Linden van den Heuvell, Phys. Rev. Lett. 61, 2099 (1988); John A. Yeazell and C. R. Stroud, Jr., Phys. Rev. Lett. 60, 1494 (1988).

[5] B. Broers, J.F. Christian, J.H. Hoogenraad, W. J. van der Zande, H. B. van Linden van den Heuvell, and L. D. Noordam, Phys. Rev. Lett. 71, 344 (1993).

[6] R. R. Jones, C. Raman, D.W. Schumacher, and P. H. Bucksbaum, Phys. Rev. Lett. 71, 2575 (1993).

[7] R. R. Jones, D. W. Schumacher, T.F. Gallagher, and P. H. Bucksbaum, J. Phys. B 28, L405 (1995).

[8] J.A. Yeazell, M. Mallalieu, J. Parker, and C. R. Stroud, Jr., Phys. Rev. A 40, 5040 (1989); J. A Yeazell and C. R. Stroud, Jr., ibid. 43, 5153 (1991).

[9] N.E. Tielking, T. J. Bensky, and R. R. Jones, Phys. Rev. A 51, 3370 (1995).

[10] Thomas F. Gallagher, Rydberg Atoms (Cambridge University Press, Cambridge, England, 1994). 